\title{
HOLLOW CYLINDRICAL LOBES WITH A HELICAL VELOCITY FIELD OF THE L1551 BIPOLAR FLOW
}

\author{
Yutaka UCHIDA ${ }^{1}$ Norio KAIFU2, Kazunari SHIBATA ${ }^{3}$, \\ Saeko S. HAYASHI 2 and Tetsuo HASEGAWA 2
}

1. Tokyo Astronomical Observatory, University of Tokyo

2. Nobeyama Radio Observatory, TAO, University of Tokyo

3. Department of Earth Science, Aichi University of Education

\begin{abstract}
Observations of the structure and the velocity field in the L1551 bipolar flow were made with the $45 \mathrm{~m}$ telescope at Nobeyama in the $115 \mathrm{GHz}{ }^{12} \mathrm{CO} \mathrm{J}=1$ - 0 line with high spatial resolution. It was found that the bipolar flow lobes have a clear hollow cylindrical structure and show evidence of a helical velocity field. They appear to rotate in the same direction as the CS disk found by Kaifu et al.(1984). The velocity of the flow in the bipolar directions increases with distance up to $\sim 3^{\prime}$ from the central object, IRS 5 . These characteristics coincide with those predicted by the magnetodynamic theory proposed by Uchida and Shibata and indicate the essential importance of the magnetic field in producing such flows and also in the star-formation process itself through the enhancement of angular-momentum loss.
\end{abstract}

\section{INTRODUCTION}

Molecular bipolar flows in or near dark clouds constitute a new category of celestial object, and several tens of them have been found after Snell et al.'s (1980) discovery of a bipolar flow in the dark cloud L1551. They have attracted attention since they are considered to be intrinsically related to the process of star-formation. They are low temperature flows ejected at supersonic velocities from the neighborhood of IR sources into two opposite directions. Observed properties of them are summarized, for example, in Bally and Lada (1983), and Lada (1985).

One of the typical examples, most suitable for a detailed examination, is the bipolar flow L1551 itself (Figure 1a), since its angular size is comparatively large and the inclination from the plane of the sky appears to be small. Observations have been made with the purpose of obtaining a detailed knowledge about the internal structure and the velocity field in it (Fridlund et al. 1984) and a higher 

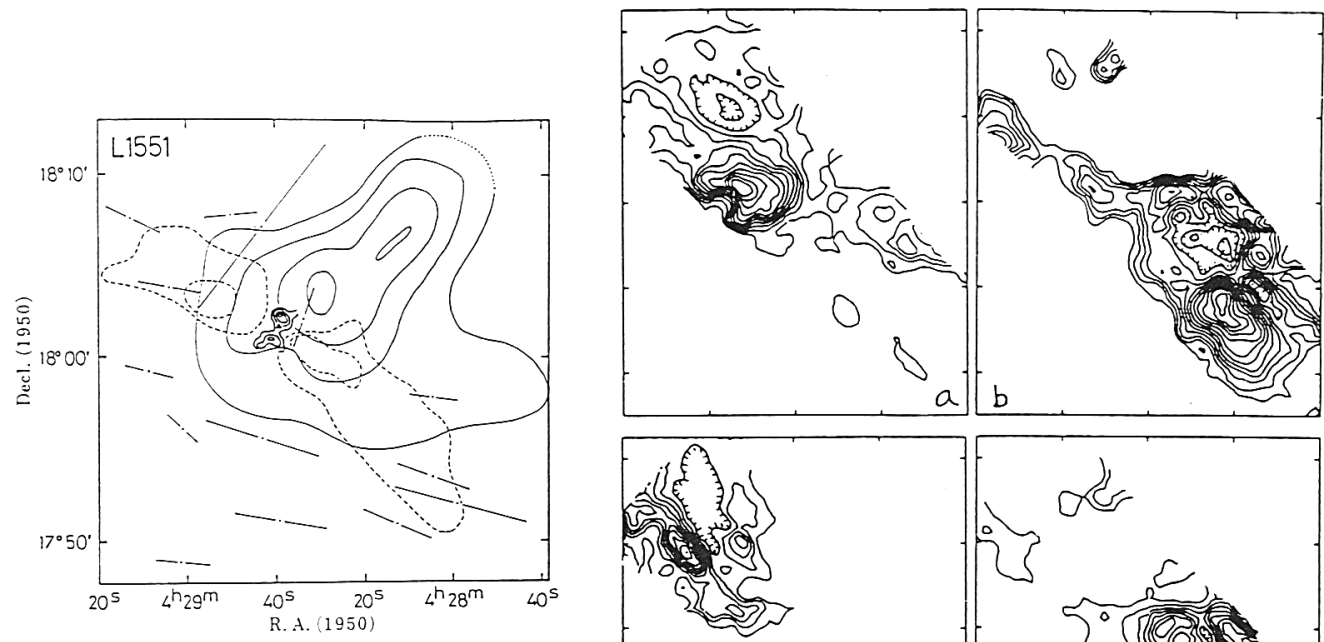

Fig. 1a: General view of the L1551 bipolar flow (Snell et al. 1980) with the CS disk around IRS 5 (Kaifu et al. 1984).
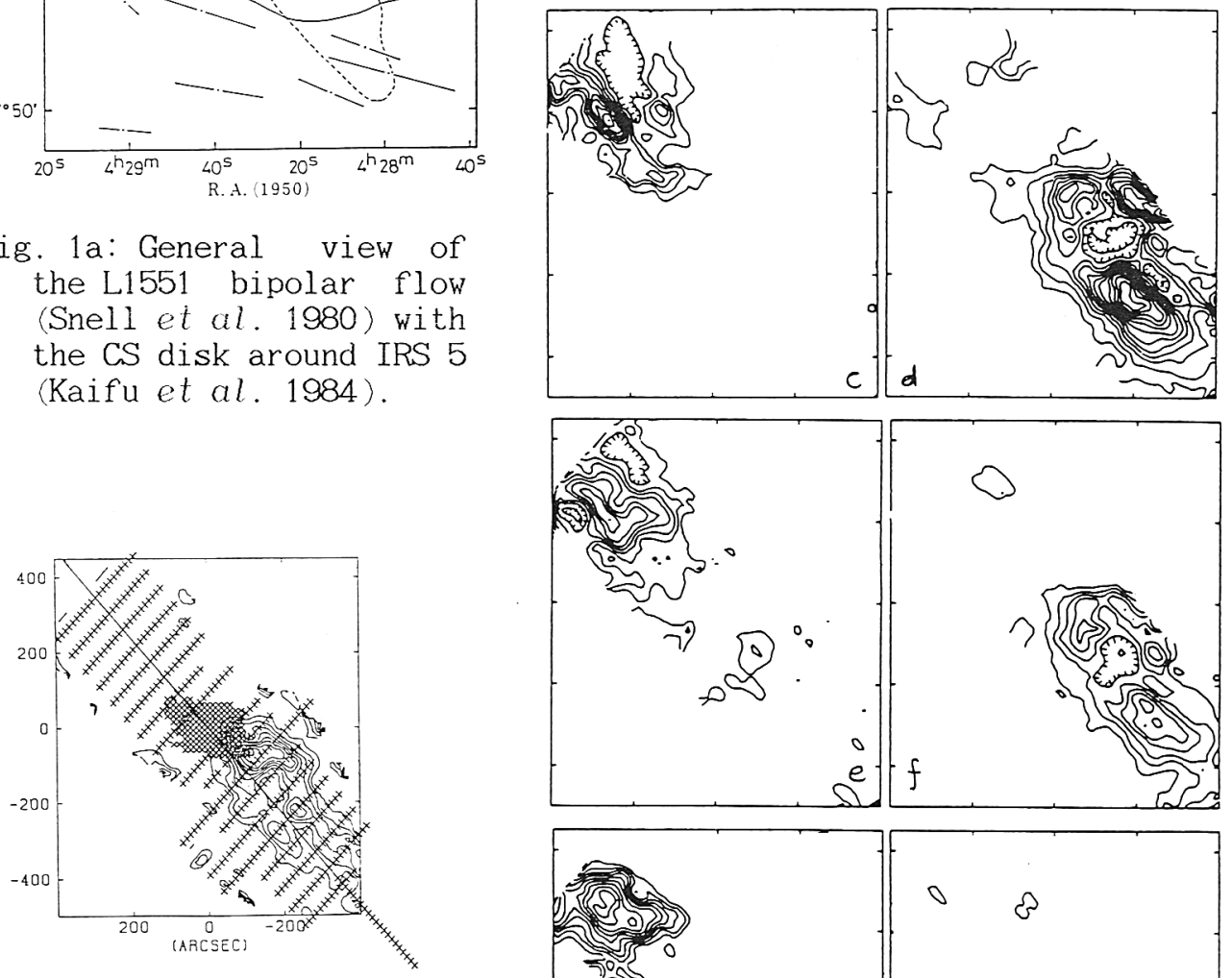

Fig. 1b: Pattern of the observed points.

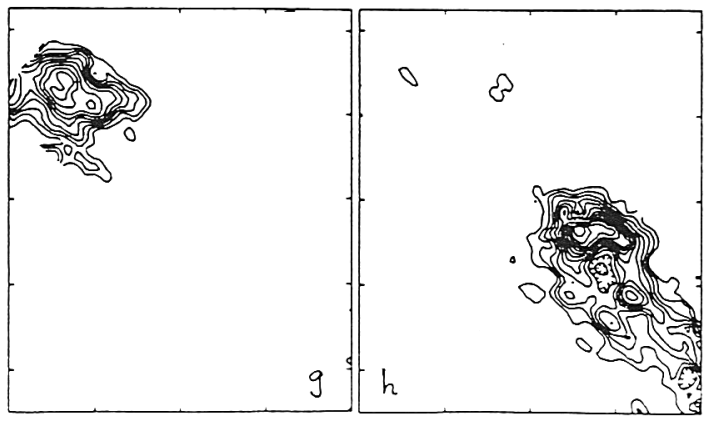

Fig. '2a through 2h: a, c, e, and $\mathrm{g}$ are red-shifted contour maps with $v_{r}=1.5 \pm 0.5,4 \pm 0.5,7.5 \pm 0.5$, and $11.5 \pm 2.5 \mathrm{~km} / \mathrm{s}$, and $b, d, f$ and $\mathrm{h}$ are blue-shifted contour maps with $\mathrm{v}_{\mathrm{r}}=-1.5 \pm 0.5,-2.5 \pm 0.5$, $-4.5 \pm 0.5$, and $-8.5 \pm 2.5 \mathrm{~km} / \mathrm{s}$, respectively. 
resolution observation was attempted by using a lunar occultation (Snell and Schloerb 1985 ). Kaifu et al. (1984) observed the central part of the lobe of the molecular flow in the $49 \mathrm{GHz}$ CS line and found a disk-like object rotating with $0.35 \mathrm{~km} / \mathrm{s}$. They also found in the 115 $\mathrm{GHz} \mathrm{CO}$ line that the flow has a larger velocity at larger distances from the disk at least in its vicinity (Kaifu et al. 1986; also Kaifu 1987 ).

In this paper, we present new results of a large-area mapping of L1551 with high spatial resolution performed at the Nobeyama Radio Observatory with the purpose of clarifying its overall internal structure and its internal velocity distribution. In section 2 the observational method is briefly described, and the results are discussed in section 3 . In section 4 we introduce an empirical model which fits these observationl results. Finally, in section 5 we discuss briefly the implication of these observations for discriminating theoretical models.

\section{OBSERVATIONS}

The observations were made in the ${ }^{12} \mathrm{CO} \mathrm{J}=1-0$ line $(115.271 \mathrm{GHz})$ with the $45 \mathrm{~m}$ telescope at the Nobeyama Radio Observatory in January and April, 1985. A detailed description of the observations will be given elsewhere (Uchida et al. 1986). With the beam size of $15^{\text {" }}$ at this frequency, it was not easy to cover densely the entire region of L1551 in the limited extent of the alloted time, and we chose the points of observation along strips across the lobes taken every 60" from the central object, as shown in Figure 1b. This sampling method was taken because one of our intentions was to examine the detailed velocity field in the flow, and in particular, to try to establish the presence of rotation of the flow lobes around their axis as predicted by Uchida and Shibata (1985b, c ). The region near IRS 5 was already observed with a 15" mesh (Kaifu et al. 1986; also Kaifu 1987), and these data were combined with the present observations to construct the entire map of the L1551 bipolar flow.

\section{OBSERVATIONAL RESULTS}

Some of the new findings obtained with our observations are described below.

\subsection{Tuning-Fork Shape Distribution of the CO Emission Intensity --- Cylindrical Shell Structure of the Molecular Flow}

Figures $\mathrm{Za}$ through $\mathrm{2h}$ show the contour maps for several velocity offsets from the local-standard-of-rest velocity of the quiescent molecular cloud, $v_{L S R}=6.7 \mathrm{~km} / \mathrm{s}$. These show ( if we trace contours of appreciable intensity level) that both the south-western lobe ( referred to as lobe-B hereafter, since it is dominantly blue-shifted) 
and the north-eastern lobe (likewise lobe-R since it is dominantly red-shifted ) have "tuning-fork" shape distributions of intensity, with voids along the axis of the flow, if we disregard inhomogeneities existing in them. These blobs of strong emission with relatively low velocity may be due to a pre-existing inhomogeneity in the region. This suggests strongly that the lobes, both lobe-B and lobe-R, have a conical-cylindrical shell structure, unless we consider the much less probable case in which the "tuning-fork" accidentally faces us. The opening angle that shell is large near the central object, and then becomes small with distance from the central object along the length of the lobes (conical-cylindrical shell).

The hollow cylindrical structure of the bipolar flows has also been suggested based on previous observations (Snell and Schloerb 1985, Kaifu et al. 1986; also Kaifu 1987), but the present observations are the first to indicate clearly with high resolution that this applies to a larger region.

3.2. Skew Structure Bridging Both Lobes in the Contour Maps at Low Velocities -..- An Indication of Rotation around the Flow-Axis

A remarkable feature to be noted in the contour maps at low velocities shown in Figures $2 a$ and $2 b$ is the skew structure. This is more clearly seen in Figure 3 in which Figures $2 \mathrm{a}$ and $2 \mathrm{~b}$ are reproduced in gray scale and in overlay. The contour with $\Delta v_{r}=(1.5 \pm 0.5)$ $\mathrm{km} / \mathrm{s}$ in lobe-R extends along the western edge of the lobe- $B$, and that with $\Delta \mathrm{v}_{\mathrm{r}}=-(1.5 \pm 0.5) \mathrm{km} / \mathrm{s}$ in lobe-B extends along the eastern edge of lobe-R, where $\Delta v_{r}$ is the velocity offset from the line center, $\mathrm{v}_{\mathrm{LSR}}=6.7 \mathrm{~km} / \mathrm{s}$ (Figure $3 \mathrm{c}$ ). This is most naturally explicable if both lobes are rotating in the same direction with a small velocity $(1 \sim 2 \mathrm{~km} / \mathrm{s})$. In other words, in the presence of the directed flow velocity, the velocity field is helical (see section 4). The direction of rotation of both lobes is the same as that of the disk around IRS 5 found by Kaifu et al. (1984) in the CS $49 \mathrm{GHz}$ line. It is obvious that in order to produce rotating lobes some efficient means of transferring angular momentum to them is needed. Indeed, such a helical velocity field has been predicted in the magnetodynamic theory of Uchida and Shibata ( $1985 \mathrm{a}, \mathrm{b}, \mathrm{c}$ ), and one of the main motivations of the present observations was to seek such features.

\subsection{Relative Location of Contours for Different Velocities -- Acceleration of the Flow with Distance}

Figures $2 \mathrm{a}$ through $2 \mathrm{~h}$ show that the ridges in the maps for larger velocity offsets systematically recede, or appear at larger distances from the central object. Moreover, it is seen that the sizes of the curved ridges for higher velocities are smaller. A most suitable interpretation of this receding contours for higher velocities is that the flow confined in the shells is accelerated with distance (see section 4). An alternate possibility suggested previously, namely, 

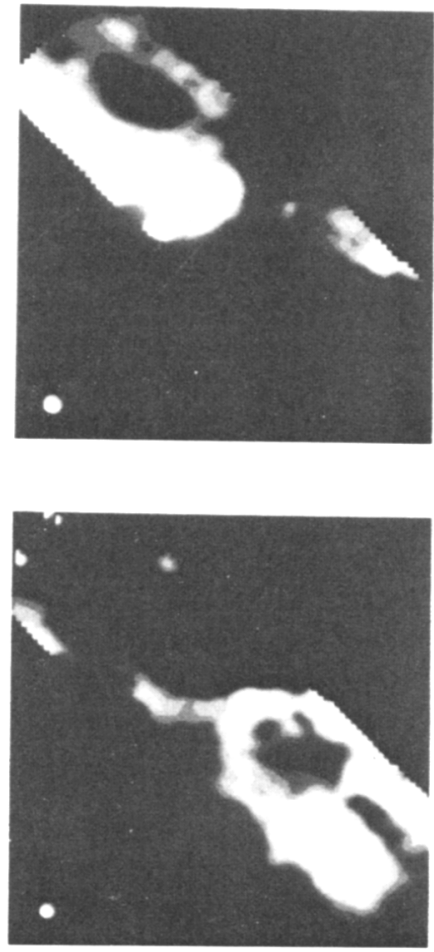

Fig. $3 a$ and 3b: Grayscale figures corresponding to Figures $2 \mathrm{a}$ and $2 \mathrm{~b}$.

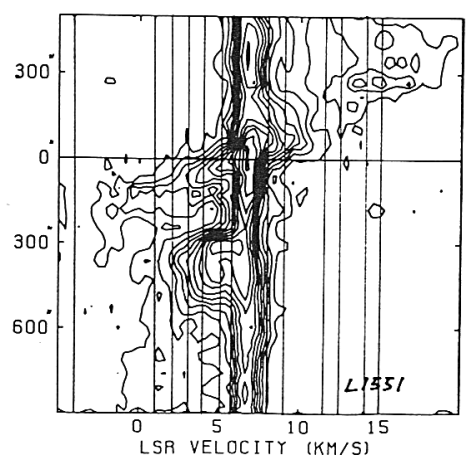

Fig. 4: P-V map along the projected axis of the flow.

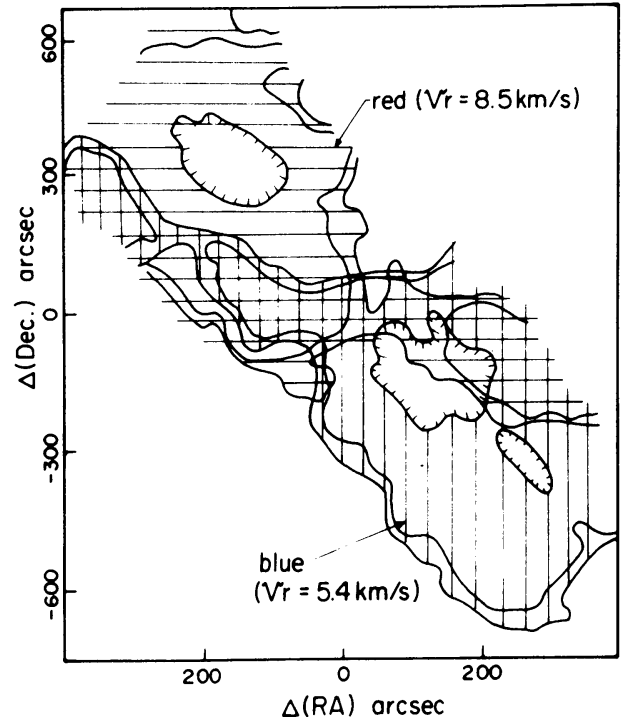

Fig. 3c: Overlay of Figures $3 a$ and $3 b$.

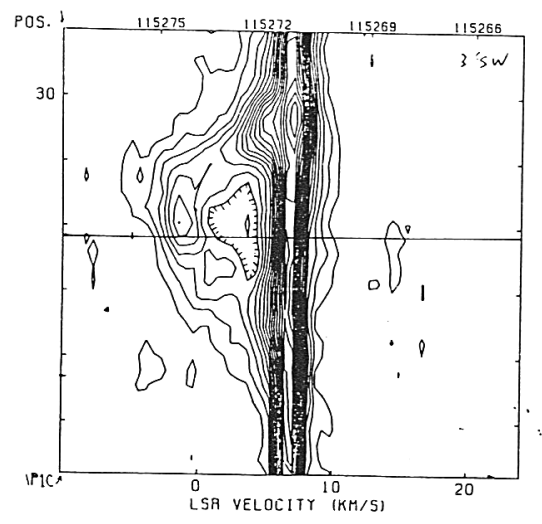

Fig. 5. P-V map along a strip across lobe-B at $3^{\prime}$ from IRS 5 . 
that the higher velocity flow occupies the inner part of the tube closer to the axis and the lower velocity flow is streaming closer to the surface of the lobe, is not very likely since the contours for higher velocities do not seem to connect themselves to the central object. Also, the fact that the position-velocity diagrams also have voids (e.g. Figure 5 ) makes such a possibility unlikely.

It is seen, however, that the acceleration of the flow takes place only up to a certain distance, and then the flow seems to be decelerated.

3.4. Position-Velocity Diagram along the Axis of the Flow in Projection ---Further Evidence for the Acceleration of the Flow with Distance

Figure 4 is a position-velocity diagram along the axis of the flow in projection. It shows that the line-of-sight velocity increases almost linearly with the distance from IRS 5 up to $3^{\circ}$, corresponding to a linear projected distance of about $0.14 \mathrm{pc}$. This is clear evidence that the acceleration of the molecular flow continues to take place at a considerable distance. The feature seems to be bent towards smaller velocities beyond this point. It is noticeable that there is another feature at a low velocity around $z \sim 350 "$, near the distance of termination of the main linear feature. This may be due to an interception of the flow by a pre-existing blob of gas which could induce a slower secondary flow. Another interpretation for this low velocity feature is that the contribution of the back-side of the shell ( for the case of lobe-B ) begins to show up since the angle of the part of the shell makes with the line-of-sight begins to deviate from $90^{\circ}$ there (see Figures 7c and 8 ). Further observations of the velocity field, specifically around the blob, will make this point clearer.

\subsection{Position-Velocity Diagrams along the Strips across the Lobes -- Evidence of Cylindrical Flow with Acceleration}

Figure 5 is a typical position-velocity diagram taken along a strip at a distance of $3^{\prime}$ from IRS 5 on lobe-B. This figure clearly shows a loop-like shape ( half-an-ellipse type shape) again with a void. We identify this half-an-ellipse type loop with flows on the cylindrical surface because it will be shown later in section 4 that the lower half of this ellipse tends to be flattened and may not be seen clearly for being embedded in the width of the strong central line under the following circumstances: (a) the relevant part of the lobe shells, the far-half of lobe-B, and the near-half of lobe-R, lie nearly perpendicularly to the line-of-sight, and (b) the flow velocity on the part of the lobe closer to the central object is smaller, or the flow is accelerated with distance along the cylindrical shell.

In summary, we found that the flow takes place on conical-cylindrical shells and is accelerated in helical paths up to a considerable distance. The direction of rotation of both lobes is the same as that of the disk around the protostar. 


\section{EMPIRICAL MODEL EXPLAINING THE OBSERVATIONAL RESULTS}

In this section, we try to construct a simple empirical model which gives features consistent with those of the observed flow described in section 3. In conformity with the observed results described in sections 3.1 and 3.5, we assume that the flow is confined on the surface of the lobes (in the following we deal with lobe-B as the representative of these two lobes), which is expressed in three-dimensions as $z=\operatorname{ar}^{\gamma}$ for simplicity. $\quad r$ and $z$ are to be measured in a common arbitrary scale. The values of $a$ and $\gamma$ to represent the observed shell shape are $a \sim 1$ and $\gamma \sim 3$. The velocity along the surface is decomposed into $\left(\mathrm{v}_{\mathrm{r}}, \mathrm{v}_{\varphi}, \mathrm{v}_{z}\right)$ and is represented by the total translational velocity $v_{t}=\left(v_{r}{ }^{2}+v_{z}^{2}\right)^{1 / 2}$ and by the rotational velocity $v_{\varphi}$. Thus, $v_{r}$ and $v_{z}$ can be expressed in terms of $v_{t}(z)$ and $v_{\varphi}(z)$ by using geometrical relations on the lobe. The geometrical situation is seen in Figure 6 and the inclination of the flow-axis from the plane of the sky is taken to be $\sim 15^{\circ}$ by the trial-and-error process performed below.

We can calculate from this model the iso-velocity contour maps and various position-velocity maps to be compared with the observational results described in sections 3.1 through 3.5, if we assume $v_{t}(z)$ and $v_{\varphi}(z)$. The line-of-sight velocities of the flow at the cross-points of the line-of-sight with the shell are calculated for the given geometry numerically. It should be noted that the position-velocity maps are two-valued, and that the contour maps are composed of those for the near- and the far-side of the conical-cylindrical shells, since we are seeing through both sides of the lobes.

\subsection{Position-Velocity Diagrams Calculated from a Simple Model}

Since we forsee a convex position-velocity diagram along the projected flow-axis if we assume a linear velocity increase with $z$, namely, $v_{t}(z)=c z$, because of the curvature of the near-side surface, we will try to reproduce the almost-linear position-velocity diagram by assuming a velocity curve concave for small $z$ and convex for large $z$ (acceleration is larger than a constant acceleration near the source, and then decreases at larger distances ). Namely, $\mathbf{v}_{\mathrm{t}}(\mathbf{z})$ having a form

$$
\mathrm{v}_{\mathrm{t}}(\mathbf{z})=\frac{1}{2} \mathrm{v}_{\mathrm{t} 0}\left[1+\tanh \left\{\left(\mathbf{z}-\mathbf{z}_{\mathrm{c}}\right) / \mathbf{z}_{\mathrm{w}}\right\}\right] \cdot\left(1+\frac{\mathrm{z}}{\mathbf{z}_{\mathrm{dt}}}\right)^{1 / 2}
$$

is used as a trial function in calculating $v_{r}(z)$ and $v_{z}(z)$. The fact that the observed position-velocity diagram along the projected flow-axis has only one branch (Figure 4) may be interpreted by considering that the relevant part of the surface on the far-side of lobe-B, for example, lies roughly perpendicularly to the line-of-sight, and therefore the other branch is embedded in the width of the strong 


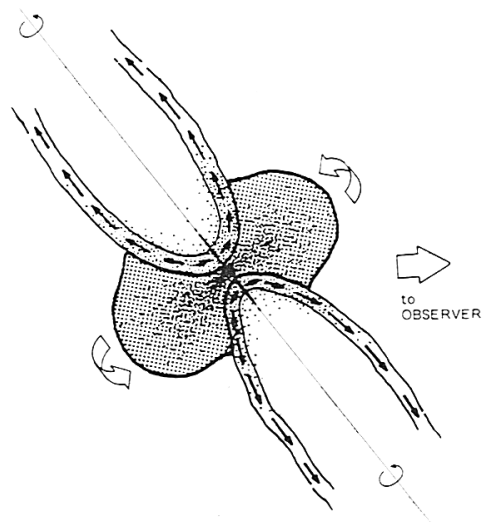

\section{Fig. 6: Schematic figure} describing the flow.

Fig. 7a: Adopted $v_{z}(z)$, $v_{r}(z)$ and $v_{\varphi}(z)$. 7b, 7c: Iso-velocity contours calculated for the emprirical model, projected on the plane of the sky. The front half and back-half of the shells are shown separetely.
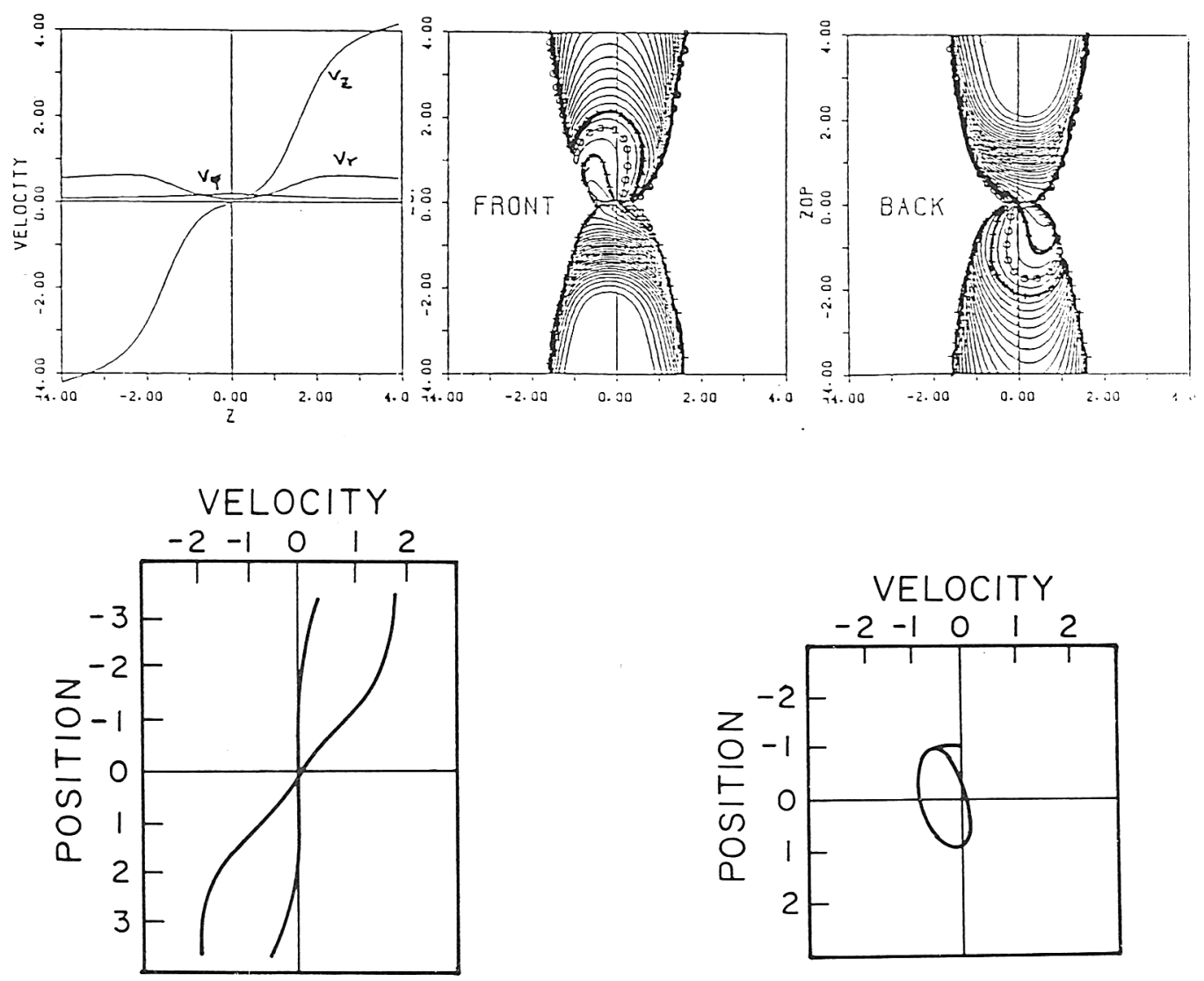

Fig. 8: Calculated P-V map along the axis of the empirical model.

Fig. 9: Calculated P-V map along a typical strip across lobe-B. 
emission of the quiescent $\mathrm{CO}$ clouds (Figure 4). This is consistent with the supposition that the inclination of the flow-axis from the plane of the sky is about $15^{\circ}$ (Cudworth and Herbig 1979).

Figure $7 a$ shows $v_{z}(z)$ and $v_{r}(z)$ calculated for the trial function which reproduces observed features well with $v_{\text {to }}=30 \mathrm{~km} / \mathrm{s}, z_{c}=1.5$, $z_{w}=0.8$, and $z_{d t}=4$ together with $v_{\varphi}(z)$ to be given later. The position-velocity diagrams along the strips across lobe-B in such a model become deformed-ellipses (Figure 9 ) which look like those loops actually observed ( Figure 5 ). The contribution from the far-side of the lobe comes from the part with smaller $z$ where $v_{t}(z)$ is smaller than that of the part on the near-side of the lobe because of the inclination of the flow-axis from the plane of the sky. Furthermore, the surface of the far-side of the shell makes almost a right angle with the line-of-sight. Figure 9 shows the case in which $v_{\varphi}(z)$ of the following trial function (shown in Figure $7 \mathrm{a}$ )

$$
\mathrm{v}_{\varphi}(\mathrm{z})=\mathrm{v}_{\varphi 0}\left(1+\frac{\mathrm{z}}{\mathrm{z}_{\mathrm{df}}}\right)^{-1 / 2}
$$

with $\mathrm{v}_{\varphi 0}=1.5 \mathrm{~km} / \mathrm{s}$ and $\mathrm{z}_{\mathrm{df}}=3$, is included. The inclusion of rotation, though small, makes an appreciable tilt of the ellipses, especially noticeable at the flattened part, as seen from Figure 9.

\subsection{Iso-Velocity Contours in the Simple Model}

The iso-velocity contours (contours of equal line-of-sight velocities ) on the projection on the sky of our simple model are calculated for the far- and near-sides of the lobes separately, and shown in Figures $7 \mathrm{~b}$ and $7 \mathrm{c}$. These show the recession of higher velocity contours toward larger $z$ together with a shrink of the size of the contours for higher velocities just as observed (section 3.3 ). The effect of rotation appears as the asymmetry with respect to the projected flow-axis at low velocities (Figures 7b and 7c ). It is indeed seen that the skew profile is successfully reproduced by the present empirical model. This shows that the skew-symmetric inter-penetration of the observed red-and blue-shifted source regions can actually be due to the effect of the rotation of both lobe-B and lobe- $R$ in the same direction as that of the rotation of the disk found by Kaifu et al. (1984). The effect is due to the predominance of the line-of-sight component of rotation velocity $v_{\varphi}$ over that of the longitudinal velocity $v_{z}$ near the disk, though $v_{\varphi}$ is of the order of 1 - $2 \mathrm{~km} / \mathrm{s}$.

The above discussion confirms that a simple empirical model, in which the flow takes place in a conical-cylindrical shell structure with an increasing longitudinal velocity and a decreasing rotational velocity with distance, can reproduce nicely the observed characteristics. The presence of a rotational velocity as well as of a gradual acceleration up to a considerable distance provides strong restrictions to the mechanisms of acceleration and collimation of the bipolar flow, and a 


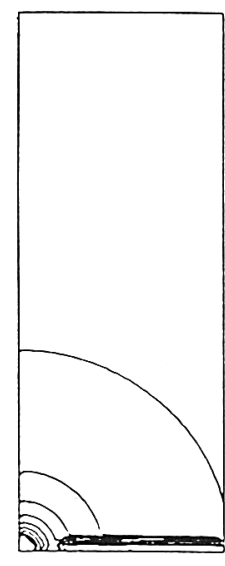

$\tau=0.07$

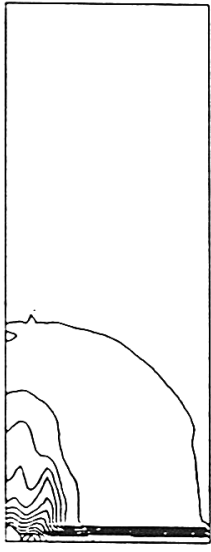

2.25

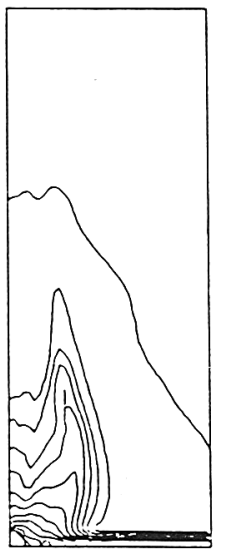

4.31

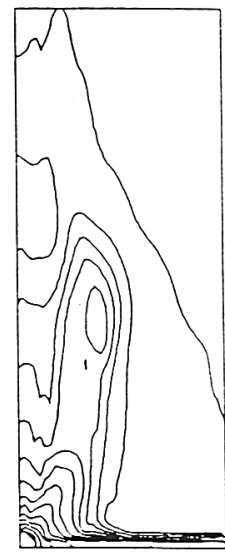

6.46

Fig. 10a: Evolution of the density contours for the Uchida-Shibata simulation model (1st quadrant only. For details, see Uchida et al. 1986 ).
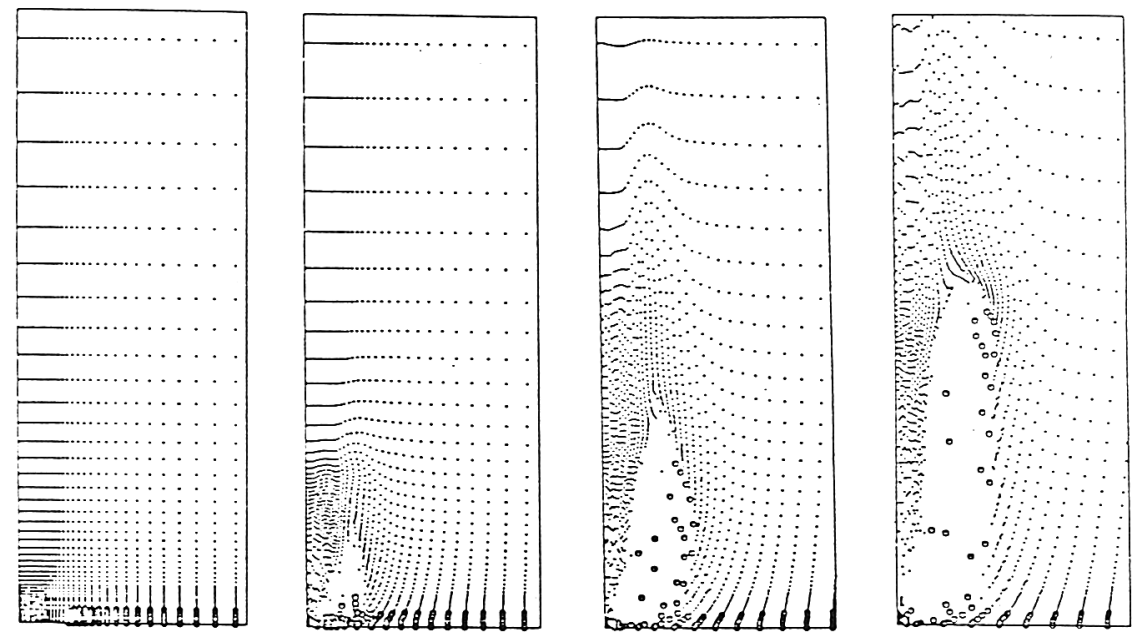

Fig. 10b: Fluid-particle motion in the $r-z$ plane in evolution. It should be noted that the front of the disturbance is ahead of the cold mass driven out from the disk (note that the number density of particle labels is not proportional to the real density ). 
brief discussion will be given on this point in the next section.

\section{DISCUSSION}

It is first noted that the motivation of the present observations was to try to see whether or not several charateristic properties of the flow predicted by the Uchida-Shibata model could be found in L1551, a typical bipolar flow. These characteristic properties predicted were: the hollow conical-cylindrical shell structure and the helical velocity field in it ( see Figures in Uchida-Shibata 1984, 1985b, c, Shibata and Uchida 1985, 1986 ) ( also Figure 10 for a longer time calculation). These predictions were indeed confirmed by our observations as described above. The hollow conical-cylindrical structure in the Uchida-Shibata theory is due to the sandglass type magnetic field pattern produced by the disk contraction. The mass in the surface region of the disk is driven out in the theory by the $j \times B$ force as the magnetic twist, which is produced and accumulated by the rotation of the disk, relaxes along the large scale magnetic field pattern. The external magnetic field is originally perpendicular to the plane of the disk and wound up into a helical field by the rotation of the disk. The flow velocity in the direction of the axis continues to increase up to a certain distance, while the rotational velocity first increases with radius due to centrifugal acceleration and then decreases due to the conservation of angular momentum. It is noted that the source of this cold helical flow is the accretion disk in the Uchida-Shibata model, in contrast with the central IR star in most of the earlier models.

The finding of rotational velocity in the flow provides a very crucial test for the previously proposed non-magnetic models since the magnetic field seems to be necessary in efficiently transferring angular momentum to collimated lobes. Indeed, the observation that the general direction of the interstellar magnetic field is parallel to the bipolar flow axis (Vrba et al. 1976), and that the IR polarization near the central source is perpendicular to it (Nagata et al. 1983), give support to the general scenario considered in the Uchida-Shibata model (Uchida and Shibata 1985b, c ). Discussion of other existing theories ( Blandford and Rees 1974, Konigl 1982, Ferrari et al. 1984 for the funnel collimation models, Sakashita et al. 1984, Okuda and Ikeuchi 1986 for the blocked snow plough models, and Pudritz and Norman 1983 for the centrifugal wind model) in relation to the present observations will be given elsewhere (Uchida et al. 1986 ).

[ Note: Uchida and Shibata (1984, 1985c ) gave an explanation for the small warm optical jet found by Mundt and Fried (1983) by means of a different but related magnetic mechanism operating near the central star.]

The authors wish to express their indebtness to Drs. M. Morimoto, H.H. Suzuki, and the staff of NRO in making observations. They also thank Mrs. H. Suzuki and Mr. Y. Siomi for their assistance in preparing the manuscript. 


\section{References}

Bally, J., and Lada, C.J. : 1983, Astrophys. J., 265, 824.

Blandford, R.D., and Rees, M.J.: 1974, Monthly Notices Roy. Astron. Soc. , 169, 395.

Cudworth, K.M., and Herbig, G. : 1979, Astrophys. J., 84, 548.

Ferrari, A., Habbal, S.R., Rosner, R., and Tsinganos, K.: 1984, Astrophys. J. Letters, 277, L35.

Fridlund, C.V.M., Sandqvist, A., Nordh, H.L. and Oloffson, G. : 1984, Astron. Astrophys. 137, L17.

Kaifu, N.: 1987, in these Proceedings.

Kaifu, N., Hayashi, S.S., Oishi, M., and Hasegawa, T., in preparation, 1986.

Kaifu, N., Suzuki, S., Hasegawa, T., Morimoto, M., Inatani, J. , Nagane, K, , Miyazawa, K., Chikada, Y., Kanzawa, T., and Akabane, K.: 1984 , Astron. Astrophys. 134, 7.

Konigl, A.: 1982, Astrophys. J., 261, 115.

Lada, C. J. : 1985, Ann. Rev. Astron. Astrophys., 23, 267.

Mundt, R., and Fried, J.W.: 1983, Astrophys. J., 274, L83.

Nagata, T., Sato, S., and Kobayashi, Y.: 1983, Astron. Astrophys. 119, L1.

Okuda, T., and Ikeuchi, S.: 1986, Publ. Astron. Soc. Japan, 38, No 2, in press.

Pudritz, R.E., and Norman, C.A.: 1983, Astrophys. J., 274, 677.

Sakashita, S., Hanami, H., and Umemura, M.: 1984, Astrophys. Space Sci. , 98, 315 .

Shibata, K., and Uchida, Y.: 1985, Publ. Astron. Soc. Japan, 37, 31.

Shibata, K. and Uchida, Y.: 1986, Publ. Astron. Soc. Japan, 38, submitted.

Snell, R.L., Loren, R.B., and Plambeck, R.L.: 1980, Astrophys. J., 239, L17.

Snell, R.L., and Schloerb, F.P.: 1985, Astrophys. J., 295, 490.

Uchida, Y., Kaifu, N., Shibata, K., Hayashi, S.S., Hasegawa, T., and Hamatake, H., 1986, in preparation.

Uchida, Y., and Shibata, K.: 1984, Publ. Astron. Soc. Japan, 36, 105.

Uchida, Y., and Shibata, K.: 1985a, in Unstable Current Systems and Plasma Instabilities in Astrophysics, ed, M. Kundu and G. Holman ( Reidel ), p. 287.

Uchida, Y., and Shibata, K.: 1985b, in The Orign of Nonradiative Heating/Momentum in Hot Stars, ed A. Underhill and A.G. Michalitsianos, NASA CP 2358 ( NASA Printing), p. 169.

Uchida, Y., and Shibata, K.: 1985c, Publ. Astron. Soc. Japan, 37, 515.

Vrba, F.J., Strom, S.E., and Strom, K.M.: 1976, Astron. J., 81, 958. 
NAKAMURA: In your model the energy source is a part of the gravitational energy of the accreted mass. What is the efficiency of the energy conversion? How much mass should be accreted to explain the total energy of the bipolar flow in L1b5l?

UCHIDA: Energy conversion efficiency is about ten percent. The required mass accretion rate is readily estimated to be $10^{-4} \mathrm{M}_{\odot} / \mathrm{yr}$ (for a flow of $10^{-5} \mathrm{M}_{\odot} / \mathrm{yr}$, Bally and Lada), and this mass losing its potential energy at around $r \sim 10^{15} \mathrm{~cm}$ will be able to provide the mechanical luminosity of the flow.

LADA: You have reported two interesting and potentially fundamental observational results that seem to imply a hydromagnetic acceleration mechanism for outflows: 1) rotation of the L1551 outflow lobes, and 2) acceleration of the outflow gas around the IR source. I would like to know if your models predict flow rotation at the ends of the lobes as well as at the origin of the lobes? Also, how far away from the flow origin do you expect to observe flow acceleration? At what point should we expect to see the tlow reach a "terminal" velocity?

UCHIDA: To the first point, the answer is yes. Namely, the rotation velocity is larger both near the disk and near the front, and smaller in between. The absolute value of the rotational velocity is much smaller than the translational velocity of this flow. The acceleration in our calculation, which is a combined effect of the time-dependence and actual acceleration, takes place at least in the first $1 / 3$ of the lobe-length. The behavior in the region further out may depend upon the density distribution out there. For example, if it comes across a higher density part (e.g. high density blob) it is decelerated and $\mathrm{B} \varphi$, and therefore $\mathrm{v} \rho$, is amplified, and so on.

BALLY: Observations show that the flow mechanical luminosity, is roughly correlated with the luminosity of the central source. 'Ihe mass of a disk may also correlate with source luminosity. In your model, how does the mass and mechanical luminosity of the outflow scale with the mass of the disk or the luminosity of the central source?

UCHIDA: I think that the luminosity of the central object is not the normal static luminosity in the period of our concern. If the luminosity is related to the liberation of potential energy of accreting material, the correlation will be something very easy to understand since the stellar luminosity as well as the mechanical luminosity of the flow may be proportional to the rate of accretion. PUDRITZ: I agree with your general point that a11 MHD wind theories should show co-rotating outflow. However, centrifugally driven wind theories such as Pudritz and Norman (1983) and Blandford and Payne (1982) predict that gas is accelerated along poloidal field near the disk, while a toroidal field developes at the Alfvén surface and beyond. If I understand your theory correctly, you predict a strong toroidal field close to the disk. I propose that the observational test to discriminate between these theories is to find on what scale the toroidal wind field is strongest. Do you agree with this?

UCHIDA: Yes, in this context, I am very interested to know the magnetic field direction in the flow region itself.

NORMAN: Do you think that the hot ionized jet component is also mag- 
netized and produced by the same mechanism?

UCHIDA: Our view about the hot tiny jets (PASJ 37,515 ) is as follows: they are also due to a magnetic mechanism but not the same as the mechanism I talked about here in relation to the cold $\mathrm{CU}$ flow. According to our view, they take place in the very vicinity of the star $\left(\mathrm{r} \sim 10^{12} \mathrm{~cm}\right.$ ) where the final fall of mass occurs under the control of a magnetic process. The fall, made possible by the effect of the magnetic field, and the mass blown oft by a recoiling shock may explain the production of the X-ray emitting region and the hot high speed ejection in a very tiny scale. 